\title{
COMPARISON OF LEFT VENTRICULAR VOLUMES AND EJECTION FRACTION ASSESSMENT BY TWO-DIMENSIONAL ECHOCARDIOGRAPHY COMPARED WITH GATED MYOCARDIAL SPECT IN PATIENTS WITH ISCHEMIC CARDIOMYOPATHY
}

\author{
Martin Hutyra*a, Tomas Skala ${ }^{\text {a }}$, Milan Kaminek ${ }^{\mathrm{b}}$, Jana Zapletalova ${ }^{\mathrm{c}}$ \\ ${ }^{a}$ Department of Internal Medicine I, Palacky University and University Hospital Olomouc, Czech Republic \\ ${ }^{b}$ Department of Nuclear Medicine, Palacky University and University Hospital Olomouc \\ c Department of Medical Biophysics, Faculty of Medicine and Dentistry, Palacky University \\ E-mail:martinhutyra@seznam.cz
}

Received: October 12, 2009; Accepted: January 22, 2010

Key words: Left ventricle ejection fraction/Enddiastolic volume/Endsystolic volume/Echocardiography/Gated myocardial SPECT

Aim. The aim of this study was to assess accuracy of echocardiography in the measurement of left ventricular ejection fraction (LVEF), enddiastolic volume (EDV) and endsystolic volume (ESV) compared with gated SPECT.

Methods. A total of 70 patients with left ventricular systolic dysfunction were enrolled to study. Median of the time interval between gated SPECT and echocardiography was 1 hour.

Results. Echocardiography was in good agreement with gated SPECT for quantification of LVEF by using Simpson's method in triplane $(\mathrm{r}=0.88, \mathrm{p}<0.001)$, biplane $(\mathrm{r}=0.83, \mathrm{p}<0.001)$ and monoplane apical four chamber projections $(\mathrm{A} 4 \mathrm{CH})(\mathrm{r}=0.71, \mathrm{p}<0,001)$. LVEF measured by SPECT $(36.6 \pm 11.5 \%)$ was non-significantly higher then triplane ( $35.9 \pm 10 \%$, median dif. $0.4 \%$ ), biplane $(35.7 \pm 10 \%$, median dif. $0.7 \%)$, A4CH monoplane $(36.6 \pm 12.2 \%$, median dif. $0.1 \%)$.

Echocardiography correlated with SPECT in quantification of EDV using triplane $(\mathrm{r}=0.8, \mathrm{p}<0.001)$, biplane $(\mathrm{r}=0.81$, $\mathrm{p}<0.001)$ and monoplane echocardiography $(\mathrm{r}=0.76, \mathrm{p}<0.001)$. EDV measured by SPECT $(201.1 \pm 72.5 \mathrm{ml})$ was significantly different and higher then triplane (174.4,5 $\pm 60.8 \mathrm{ml}$, median dif. 22.8), biplane (170.9 \pm 58.4 , median dif. 28 $\mathrm{ml})$ and monoplane echocardiography (173.7 \pm 59.3 , median dif. $85.7 \mathrm{ml})$.

Echocardiography correlated with SPECT in quantification of ESV by triplane $(\mathrm{r}=0.87, \mathrm{p}<0.001)$, biplane $(\mathrm{r}=0.86$, $\mathrm{p}<0.001)$ and monoplane echocardiography $(\mathrm{r}=0.83, \mathrm{p}<0.001)$. ESV measured by SPECT $(133.1 \pm 64.3)$ was significantly different then triplane (115.1 \pm 52.1 , median dif. $17 \mathrm{ml})$, biplane $(113.2 \pm 51.5$, median dif. $15.5 \mathrm{ml})$ and $\mathrm{A} 4 \mathrm{CH}$ monoplane echocardiography (112.5 \pm 48.8 , median dif. $17.2 \mathrm{ml})$.

Conclusion. Echocardiography is a valid tool for LVEF assessment and systematically underestimates LV volumes compared with gated SPECT in patients with LV systolic dysfunction.

\section{INTRODUCTION}

An accurate assessment of ejection fraction (LVEF) and volumes of left ventricle plays a key role in diagnostic of heart diseases, optimal treatment strategy selection and has an important prognostic potential in light of following cardiovascular events prediction mainly in patients with left ventricle systolic dysfunction ${ }^{1,2}$.

LVEF is a widely used parameter in clinical praxis. It is also important in light of an optimal treatment strategy selection for instance in patients with a heart failure before implantation of biventricular pacemaker, implantable cardioverter-defibrillator or an optimal revascularization strategy selection. The effect of quite a number of such treatment procedures in patients with LV systolic dysfunction is evaluated in terms of reverse remodeling induction evaluated mainly by assessment of LVEF and the change of $\mathrm{LV}$ volumes.

Endsystolic and enddiastolic LV volumes represent basic measured parameters from which LVEF is assessed using a simple calculation. The evaluation of endsystolic and enddiastolic LV volumes brings quantitative information about the level of LV remodeling and about possibility of its reversibility after onset of treatment of a given myocardium disease. Moreover, an assessment of LV volumes brings in important prognostic information in patients after myocardial infarction, cardiosurgical correction of valvular heart diseases and after quite a number of other therapeutic interventions ${ }^{3-5}$.

Radionuclide methods focused on LVEF assessment were until recently based mainly on radionuclide ventriculography. This method is highly reproducible and easily accomplishable with relatively low expenses. Gated SPECT is usable also in patients with a poor echocardiographic image quality with an inadequate endocardial contours delineation for a good-quality assessment of regional kinetics of LV (up to $30 \%$ of patients), it is independent on subjective assessment and provides a complex information about global and regional LV function, regional perfusion and possibly about particular LV segments metabolism. 
The magnetic resonance (MR) of heart is a potentially very promising method, that besides a detailed LV function and morphology evaluation can be used for direct visualization of myocardial scar, its localization and estimation of its range using post-contrast saturation (delayed enhancement phenomenon). Compared to radionuclide methods MR is not affected with attenuation artifacts and patient is not exposed to radiation.

There are many studies published so far comparing different echocardiography approaches in measuring LV EF and volumes with gated SPECT of myocardium. The majority of papers published on this topic compared these methods performed after a severe time interval in which LV volumes and EF can vary depending on many circumstances. Additionally there is a lack of evidence for a precise measurement of LVEF and volumes mainly in a group of patients with LV systolic dysfunction in ischemic cardiomyopathy. That is why we aimed our work at a comparison of accuracy of LVEF and volumes measurement using 2D echocardiography and gated SPECT of myocardium performed in tight sequence. Gated cardiac SPECT was considered a gold standard in patients with ischemic cardiomyopathy with a majority of patients with LV systolic dysfunction. The aim was the comparison of gated SPECT and echocardiography form the viewpoint of their interchangeability.

\section{PATIENT GROUP AND METHODOLOGY}

70 consecutive patients were enrolled to study, all of them examined at Department of Internal Medicine I., University Hospital Olomouc during 10/04-12/06 within the scope of a prospective follow-up of patients with ischemic cardiomyopathy indicated to cardiosurgical revascularization based on result of coronarography.

Every patient had an optimalized treatment of ischemic heart disease and heart failure and was in a heart failure functional class NYHA I-III. A dominant part of the evaluated group had a presurgery echocardiographically verified LV systolic dysfunction defined by LVEF < $50 \%$ ( $n=65 \%$ of the whole group of patients).

All patients within the frame of a complex examination of LV viability and myocardial function after signing a written consent underwent presurgery echocardiography, gated SPECT of myocardium and in case of no contraindication also MR of heart. All examinations were performed in a tight sequence, just one after the other, all of them in one single center. All examinations were assessed offline blindly without knowing results of other performed examination methods.

\section{Echocardiography}

70 patients were enrolled into study, $70 \%(n=49)$ with an optimal echocardiography image quality, defined by a complete delineation of endocardial contours in the whole range of $\mathrm{LV}$ during whole heart cycle. $30 \%$ of patients $(n=21)$ had a good 2D-echocardiography image quality, but with need of a minimal endocardial border extrapolation during tracing of LV area in apical projections in the course of LV volumes assessment. Echocardiography was performed just after gated SPECT of myocardium with a median of time interval between examinations of 1 hour.

Echocardiographic examinations were performed on GE Ultrasound Vivid 7 (GE Healthcare Technologies, Waukesha, Wisconsin, USA) equipped with a multifrequency ultrasound probe usable for all Doppler aplications including quantitative tissue velocity imaging (TVI) analysis and echocontrast, velocity profiles, strain/strain rate profiles, amplitude analysis, anatomic M-mode with a possibility of a complete full-fledged analysis on archived sequences. The evaluation of echocardiographic findings was performed off-line in an environment of archival program EchoPAC 7 Option (verion BT 06) blindly without knowledge of patient's clinical status and results of other examinations. The resting examination with an obligatory synchronous registration of 1 ECG lead was performed standardly from parasternal projections on long and short axis of LV and from apical 2 and 4 chamber projections. Endsystolic and enddiastolic volumes were assessed from a 2-dimensional image (B-mode) by endsystolic and enddiastolic planimetry of endocardial border of LV performed in undermentioned echocardiographic projections ${ }^{17}$.

LV volumes and EF were assessed using Simpson's disc method from 3 apical projections on LV long axis, apical 4-chamber projection first, followed by apical 2-chamber and 3-chamber projections. All parameters were assessed as averages from 3 measurements of 1-cycle recordings during stable sinus rhythm. In case of atrial fibrillation ( $n=7,10 \%$ of all examined patients) the particular parameters were obtained by averaging from heart cycles with identical R-R intervals (differences between evaluated R-R intervals $<5 \mathrm{~ms}$ ). The single measured echocardiographic parameters were triplanar, biplanar (apical 2-chamber and apical 4-chamber projections) and monoplanar (apical 4-chamber projection) LV volumes and EF.

\section{Gated technetium-99m sestamibi (MIBI) single photon emission computed tomography (SPECT)}

All patients underwent 1-day rest imaging with nitrate administration: $8 \mathrm{mCi}$ (296 MBq) Tc-99m-MIBI was injected at rest, with SPECT imaging performed 1 hour after injection. Three hours later, $24 \mathrm{mCi}$ ( $888 \mathrm{MBq}$ ) Tc99m-MIBI was injected after sublingual nitrate administration 1 hour before SPECT imaging.

Gated SPECT imaging ( 64 projections from the $45^{\circ}$ right anterior oblique projection to the $45^{\circ}$ left posterior oblique projection, 8 frames/cycle) was performed using a 2-detector gamma camera (ECAM, Siemens, Erlangen, Germany) equipped with a low-energy, high-resolution parallel-hole collimators. Myocardial perfusion images were analyzed visually and quantitatively on computergenerated polar maps using automated, commercially available software 4D-MSPECT package (University of Michigan, Ann Arbor, MI, USA).

Gated single photon emission computed tomography rest left ventricular ejection fractions (LVEF) and left ventricular enddiastolic/endsystolic volumes (EDV/ESV) were obtained using software 4D-MSPECT. 


\section{Statistical analysis}

Statistical analysis was performed on statistical software SPSS for Windows 10.0 (SPSS Inc., Chicago, USA). The results of echocardiographic measurements were compared to gated SPECT as a reference method.

Parameters (LVEF, EDV and ESV) measured using echocardiography and gated SPECT were compared using Wilcoxon test (median difference, 95\% confidence interval). A linear correlation analysis (Pearson correlation coefficient $r$ ) was performed. Altman-Bland analysis (differences average, average +1.96 * standard deviation, average $-1.96 *$ standard deviation, bias between two methods, upper and lower $95 \%$ limit of agreement) was used to assess differences and match between both methods. Values of p0.05 were considered statistically significant.

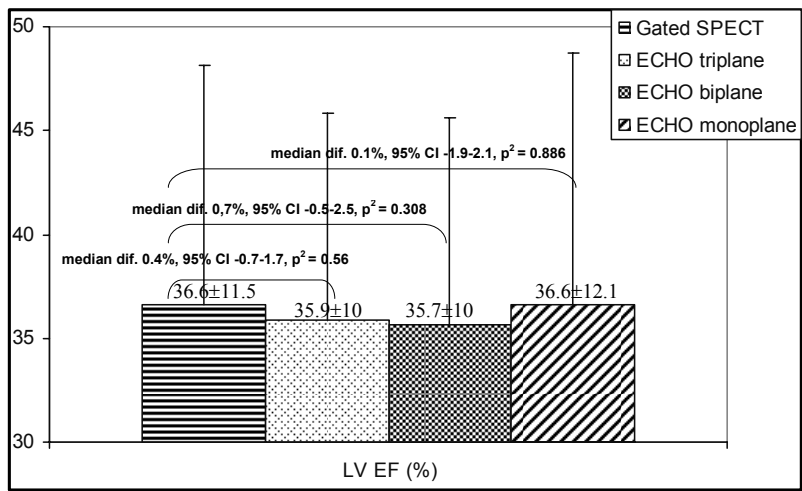

Fig. 1. Average values of LVEF with standard deviations and median differences of LVEF measurement between individual methods.
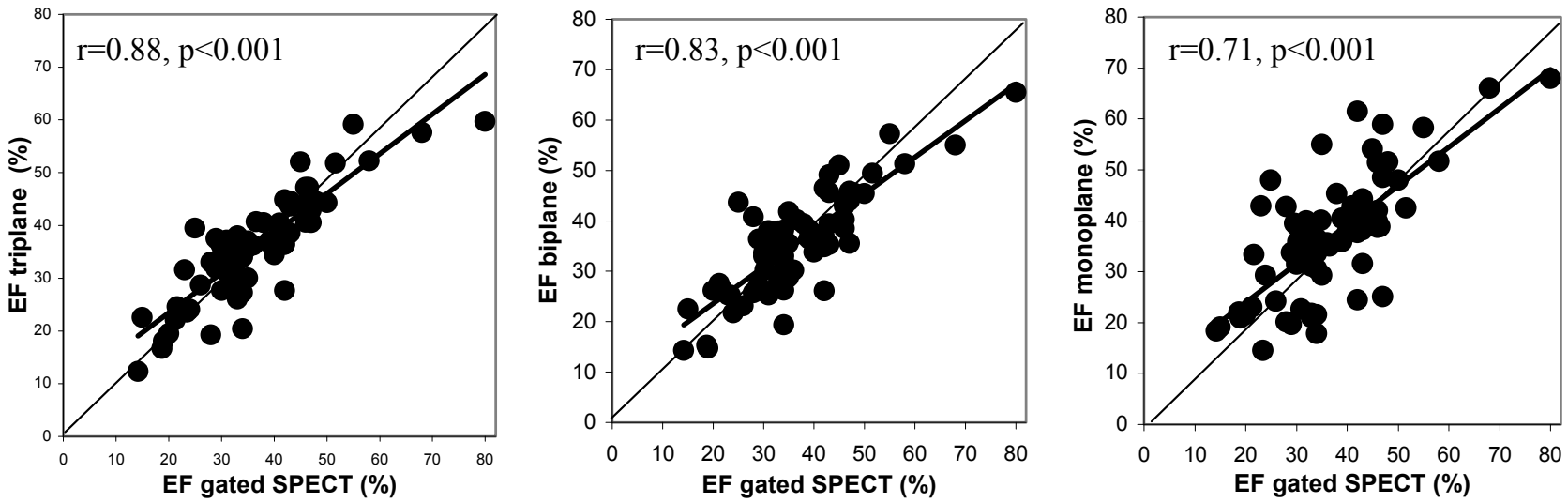

Fig. 2. Correlations of LVEF measurements using different echocardiographic approaches and measurements by gated SPECT of myocardium.

\section{RESULTS}

\section{Comparison of LV ejection fraction}

LVEF measured by SPECT (36.6 $\pm 11.5 \%)$ was slightly higher then triplane $(35.9 \pm 10 \%$, median dif. $0.4 \%, 95 \% \mathrm{CI}$ $\left.-0.7-1.7, z=0.59, p^{2}=0.56, p=N S\right)$, biplane $(35.7 \pm 10 \%$, median dif. $\left.0.7 \%, 95 \% \mathrm{CI}-0.5-2.5, \mathrm{z}=1.02, \mathrm{p}^{2}=0.308, \mathrm{p}=\mathrm{NS}\right)$, A4CH monoplane (36.6 $\pm 12.2 \%$, median dif. 0,1\%, 95\% CI $\left.-1,9-2,1, z=0,14, p^{2}=0,886, p=N S\right)$. Differences between LVEF measured using gated SPECT and each echocardiographic approach were not statistically significant. The average values of LVEF with standard deviations and average differences between each method are transparently depicted in graph 1 (Fig. 1).

Echocardiography was in good agreement with gated SPECT for quantification of LVEF using Simpson's rule in triplane $(r=0.88, p<0.001)$, biplane $(r=0.83, p<0.001)$ and monoplane apical four chamber projections $(\mathrm{A} 4 \mathrm{CH})$ $(\mathrm{r}=0.71, \mathrm{p}<0.001)$ (Fig. 2).

Bland-Altmann measurements agreement analysis between particular methods demonstrated best agreement in LVEF between gated SPECT and triplane

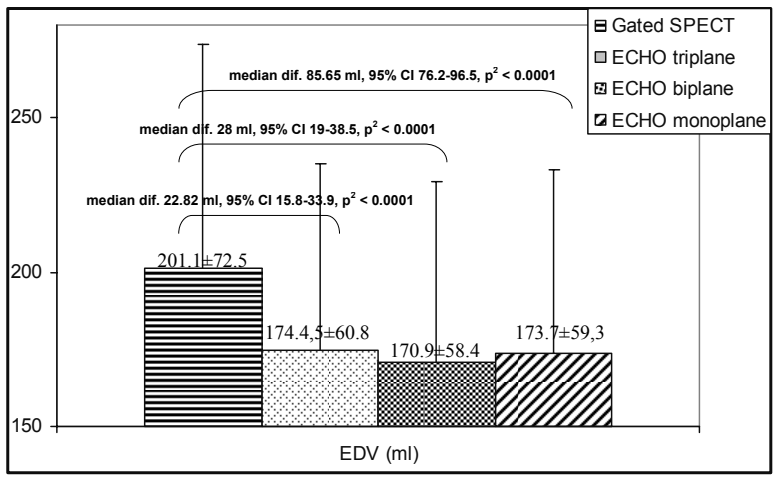

Fig. 3. Bland-Altman measurement agreement analysis of measurement of LVEF by gated SPECT of myocardium and individual echocardiographic approaches - histogram of differences. (The limits in graph are an average of differences (SPECT-ECHO) and average +1.96 . SD, average -1.96 SD respectively, standard deviation is a deviation of differences). 


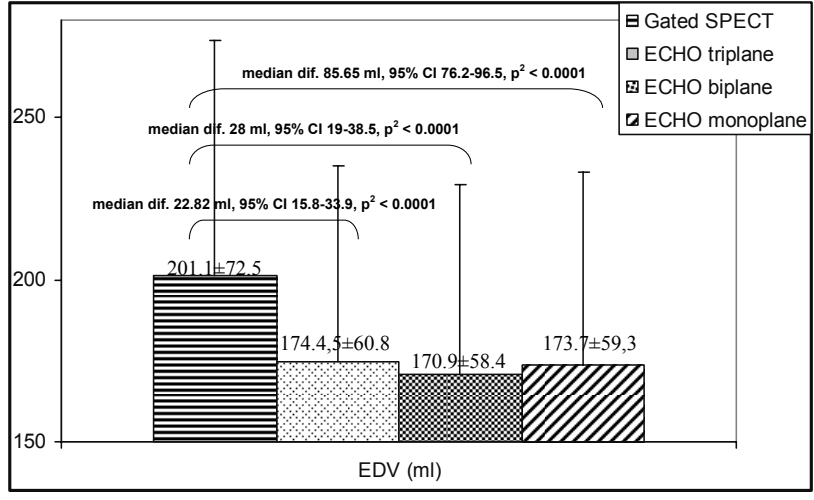

Fig. 4. Average values of EDV with standard deviations and median differences of LVEF measurement between individual methods.

echocardiography with the finding of bias $-0.4 \%$ and $95 \%$ of lower and limit of agreement in $-11.7-+10.7$ interval. Agreement between gated SPECT and biplane echocardiography measuring LVEF was very similar to the triplane method (bias $-0.7 \%, 95 \%$ lower and upper limit of agreement -13.4 - +11.7). The worse agreement with LVEF measured using SPECT of myocardium was in monoplane echocardiography (bias $-0.8 \%, 95 \%$ lower and upper limit of agreement $-17.2-+17.3 \%$ ) (Fig. 3).

\section{Comparison of LV enddiastolic volumes}

EDV measured by SPECT $(201.1 \pm 72.5 \mathrm{ml})$ was significantly different and higher then triplane $(174.4,5 \pm 60.8$ $\mathrm{ml}$, median dif. $22.8 \mathrm{ml}, 2$, 95\% CI 15.75-33.9, $\mathrm{p}<0.001$ ), biplane $(170.9 \pm 58.4 \mathrm{ml}$, median dif. $28 \mathrm{ml}, 95 \% \mathrm{CI}$ $19-38.5, \mathrm{p}<0.001)$ and monoplane echocardiography (173.7 $\pm 59.3 \mathrm{ml}$, median dif. $85.7 \mathrm{ml}, 95 \%$ CI 76.2-96.45, $\mathrm{p}<0.001)$. Differences between EDV measured using gated SPECT and each particular echocardiographic approach were statistically significant. The average values of EDV with standard deviations and average differences between each method are depicted in graph 4 (Fig. 4). Echocardiography correlated with gated SPECT in quantification of EDV using triplane $(\mathrm{r}=0.8, \mathrm{p}<0.001)$, biplane $(\mathrm{r}=0.81, \mathrm{p}<0.001)$ and monoplane echocardiography $(\mathrm{r}=0.76, \mathrm{p}<0.001)$ (Fig. 5).

Bland-Altmann measurement agreement analysis between each methods demonstrated best agreement in assessment of EDV between gated SPECT a triplane echocardiography (bias $26.7 \mathrm{ml}, 95 \%$ lower and upper limit of agreement 111.7 - $-58.4 \mathrm{ml}$ ). Agreement between gated SPECT and biplane echocardiography in measuring EDV was very similar to triplane method (bias $30.1 \mathrm{ml}$, 95\% upper and lower limit of agreement $114--53.7 \mathrm{ml}$ ). The worse agreement with LVEF measured using SPECT of myocardium was using monoplane echocardiography (bias $88.6 \mathrm{ml}, 95 \%$ upper and lower limit of agreement 175.2 - $2.1 \mathrm{ml}$ ) (Fig. 6).

\section{Comparison of $L V$ endsystolic volumes}

ESV measured by SPECT $(133,1 \pm 64,3 \mathrm{ml})$ was significantly different and higher then triplane $(115.1 \pm 52.1$
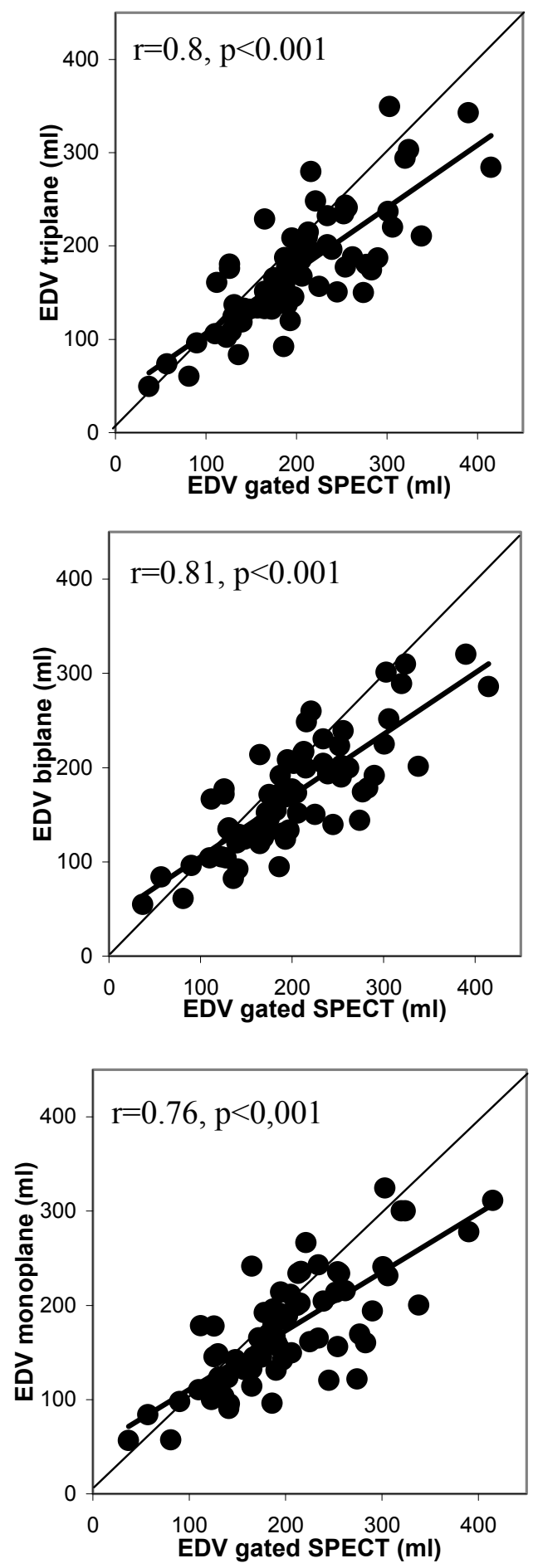

Fig. 5. Correlation of EDV measurement using different echocardiographic approaches and gated SPECT of myocardium.

ml, median dif. $17 \mathrm{ml}, 95 \% \mathrm{CI} 11.5-23.5, \mathrm{z}=4.67, \mathrm{p}<0.001$, $\left.\mathrm{p}^{2}<0.0001\right)$, biplane $(113.2 \pm 51.5 \mathrm{ml}$, median dif. $15.5,95 \%$ CI 8.4-22.5, $\left.\mathrm{z}=7.25, \mathrm{p}<0.001, \mathrm{p}^{2}<0.0001\right)$ and $\mathrm{A} 4 \mathrm{CH}$ monoplane echocardiography $(112.5 \pm 48.8 \mathrm{ml}$, median dif. 17.2 ml, 95\% CI 10.2-25.6, $\mathrm{z}=4.45, \mathrm{p}<0.001$, $\left.\mathrm{p}^{2}<0.0001\right)$. Differences between EDV measured using gated SPECT and all echocardiographic approaches were statistically significant. The average values of ESV 

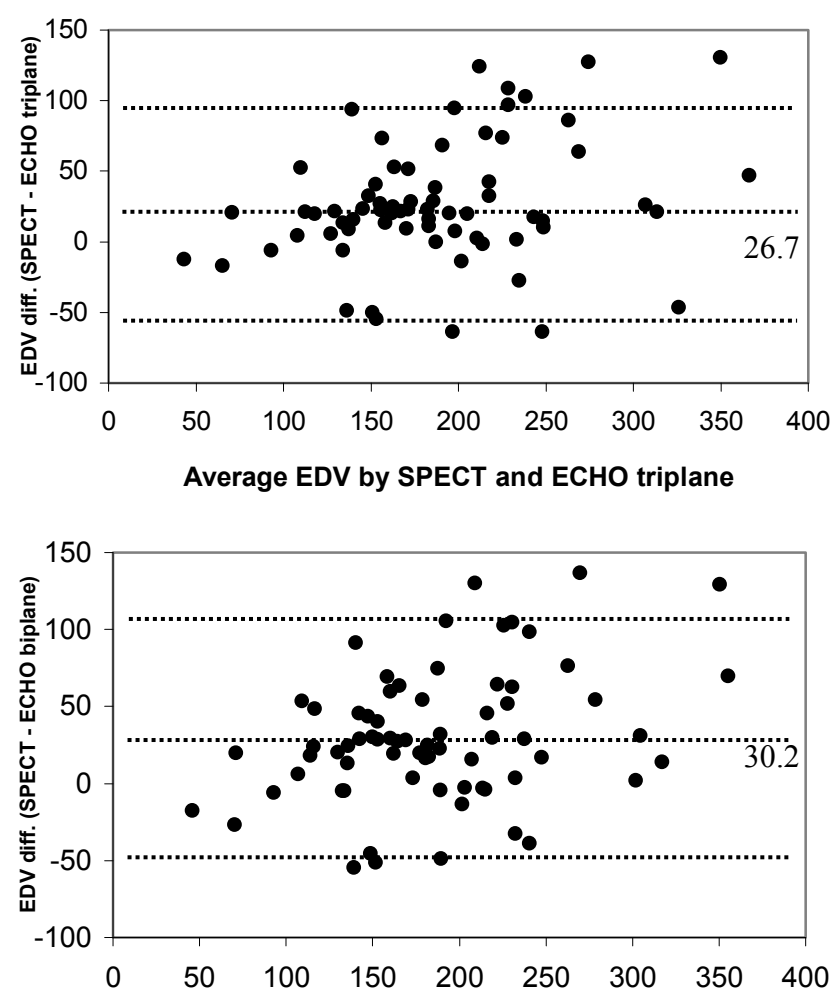

Average EDV by SPECT and ECHO biplane

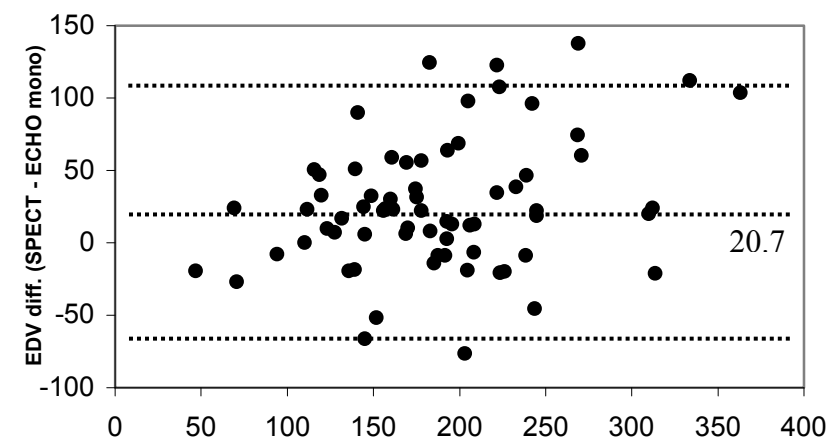

Average EDV by SPECT and ECHO monoplane

Fig. 6. Bland-Altman measurement agreement analysis of EDV measurement by gated SPECT of myocardium and by different echocardiographic approaches. - histogram of differences. (The limits in graph are an average of differences SPECTECHO) and an average $+1.96_{*},-1.96_{*} \mathrm{SD}$ respectively, standard deviation is a deviation of differences).

with standard deviations and average differences between each particular method are depicted in graph 4 (Fig. 7). Echocardiography also correlated with gated SPECT in quantification of ESV using triplane $(r=0.87, p<0.001)$, biplane $(\mathrm{r}=0.86, \mathrm{p}<0.001)$ and monoplane echocardiography $(\mathrm{r}=0.83, \mathrm{p}<0.001)$ (Fig. 8$)$.

Bland-Altmann measurement agreement analysis between each methods demonstrated best agreement in assessment of ESV between gated SPECT and triplane echocardiography (bias $18 \mathrm{ml}, 95 \%$ upper and lower limit of agreement 81.2 - $-45.2 \mathrm{ml}$ ). Agreement between gated SPECT and biplanar echocardiography in measuring

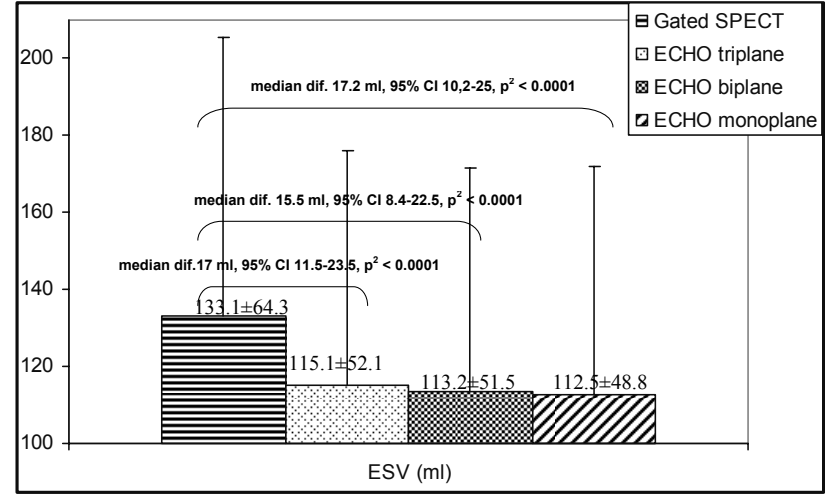

Fig. 7. Average values of ESV with standard deviations and median differences of individual methods.
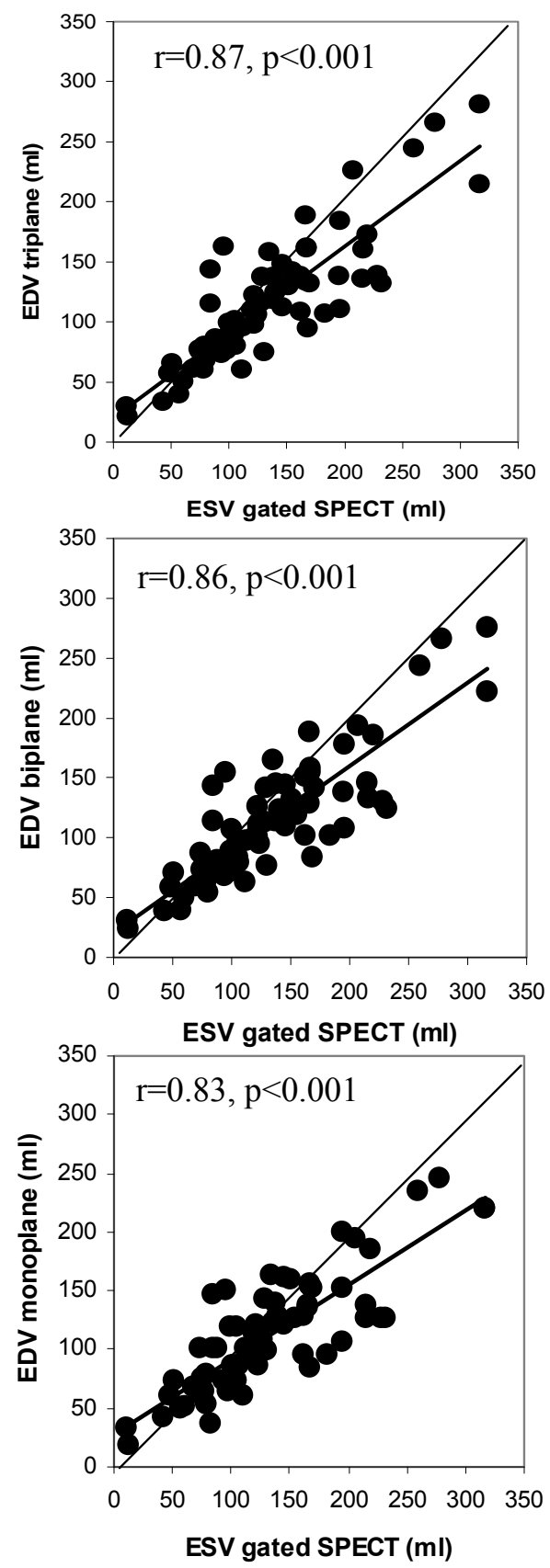

Fig. 8. Correlations of ESV measurements using different echocardiographic approaches with gated SPECT of myocardium. 

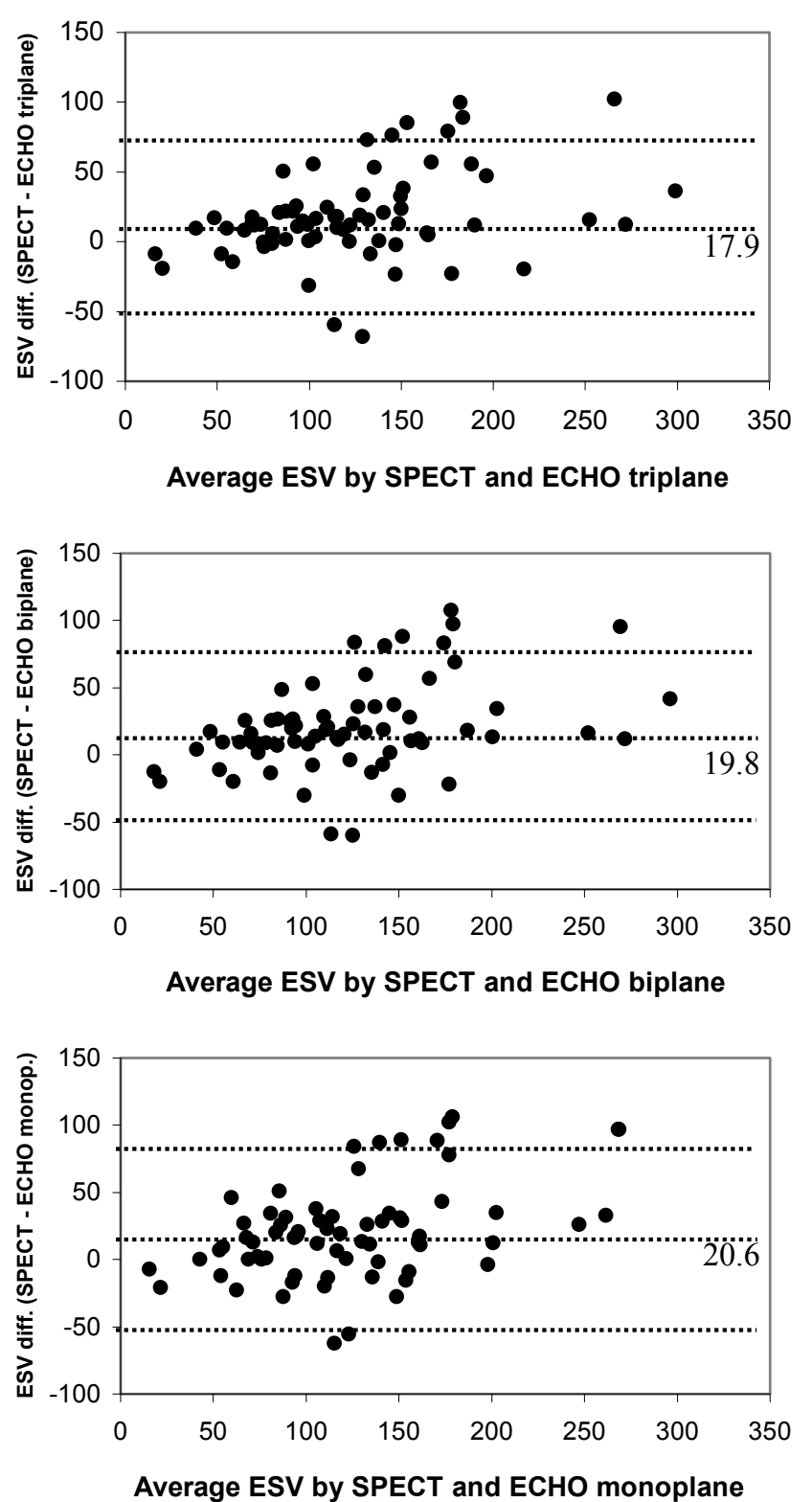

Fig. 9. Bland-Altman measurement agreement analysis of ESV measurement between gated SPECT of myocardium and individual echocardiographic approaches - histogram of differences. (The limits in graph are an average of differences SPECTECHO) and an average $+1.96_{*}$, average $-1.96_{*} \mathrm{SD}$ respectively, standard deviation is a deviation of differences).

LVEF was very similar to triplane method (bias $19,9 \mathrm{ml}$, 95\% upper and lower limit of agreement $85.2--45.4 \mathrm{ml}$ ). The worse agreement of LVEF measured using SPECT of myocardium was in monoplane echocardiography (bias $20.6 \mathrm{ml}, 95 \%$ lower and upper limit of agreement 90.8 $-49.7 \mathrm{ml}$ ) (Fig. 9).

\section{DISCUSSION}

Taking into account the important prognostic information and key therapeutic applications of one-time measurement of LV volumes and especially LVEF it is important to assess the diagnostic accuracy of currently available imaging methods for measuring these parameters. Especially it is important to accurately assess LV volumes and EF in patients with LV systolic dysfunction because measurement of these parameters plays a key role in therapeutic management, for instance in indication for a resynchronization therapy of heart failure or implantation of cardioverter-defibrillator. These therapeutic procedures are relatively very expensive but in selected patients, there is a very good cost-benefit ratio. A one-time measurement of LV volumes and EF can be severely affected by quite a number of non-constant influences that can possibly distort the examination's result. Among objective factors that are able in a very short time horizon to fundamentally modify the result of a one-time echocardiographic measurement of volumes and especially LVEF belong mainly tachyarrhythmias (e.g. atrial fibrillation with fast ventricular response with a markedly variable heart cycle length), bad image quality with an inadequate delineation of endocardial contours, acute LV preload and afterload changes. Examining physician's experience with knowledge of each particular echocardiographic approaches limitation plays also an important role in an accurate assessment of LV volumes and EF. It is a reality that about $30 \%$ of patients indicated for echocardiography have a poor image quality and an objective assessment of $\mathrm{LV}$ volumes and $\mathrm{EF}$ is not possible.

If an accurate quantification of these parameters is needed it is possible to enhance delineation of endocardial contours by use of an echocontrast agent infiltrating $\mathrm{LV}$ or to indicate $\mathrm{LV}$ volumes and $\mathrm{EF}$ assessment by some alternative imaging method such as gated SPECT of myocardium. Despite a relatively big amount of studies correlating LVEF assessment by echocardiography and gated SPECT there is a limited amount of studies concerning a specifically selected group of patients with ischemic cardiomyopathy, systolic LV dysfunction and top of that with an LVEF measurement by all mentioned methods performed on the instant just one after the other ${ }^{16}$.

A correlation analysis comparing echocardiographic measurement of LVEF with gated SPECT showed in our group of patients an excellent agreement in echocardiographic triplane LVEF measurement and gated SPECT. Correlations of biplanar and mainly monoplanar LVEF measurements were weaker compared to triplanar method. The strength of correlation expressed by Pearson's correlation coefficient was comparable to data in available literature with values of $\mathrm{r}=0.88$ in triplane method and $\mathrm{r}=0.71$ in monoplane method ${ }^{6,16}$. In other studies there was nevertheless found an even weaker correlation ( $r=0.68$ in a study by Cwajg, $r=0.62$ in a study by Mohan) between assessment of LVEF using 2D-echocardiography and gated-SPECT of myocardium. This finding can be due to a smaller amount of patients included in study ( $\mathrm{n}=16$ 
in a study by Mohan) and to a long time interval between individual examinations. This time interval is usually approximately 15 days long but in some studies, there has been a time interval of almost a month ${ }^{7,8,16}$.

In our study, the median of time interval between gated-SPECT of myocardium and echocardiography was 1 hour. Such a short time interval between individual examinations impedes an influence of some objective factors capable of influencing LV function (e.g. LV afterload and preload changes). In a retrospective analysis of data from 201 patients comparing accuracy of LVEF measurement echocardiography slightly but nevertheless statistically significantly overestimated LVEF compared to gated-SPECT of myocardium (49\% vs. $47 \%$ by gatedSPECT) ${ }^{9}$.

Concerning a comparison of accuracy of LVEF measurement using 2D-echocardiography and gated-SPECT of myocardium in a group of patients with systolic LV dysfunction the results of LVEF measurement agreement using abovementioned methods were in our work comparable with the study by Berk where evaluating a group of patients with an average LVEF of $29 \%$ a correlation coefficient of $r=0.72$ was found ${ }^{10}$.

This value is comparable with correlation of LVEF assessment using monoplane echocardiography and gatedSPECT of myocardium in our study. LVEF assessment using biplane or triplane Simpson's method seems according to our results as a more accurate alternative to the monoplane method. Though we did not find a statistically significant difference between average values of LVEF assessed by individual echocardiographic approaches compared to gated-SPECT of myocardium the Bland-Altman measurement methods agreement analysis demonstrated a relatively high fluctuation of differences in LVEF parameter mainly between monoplane echocardiography and gated-SPECT of myocardium. Limit of agreement between individual echocardiographic approaches is broadening the more the less echocardiographic projections are used for LVEF calculation. This finding supports our opinion that a one-time monoplane LVEF assessment is not entirely accurate and for clinical purposes unsatisfactory and that LVEF should be assessed by at least biplane echocardiography ${ }^{17}$.

This conclusion can be reasoned by the fact that in a group of patients with ischemic cardiomyopathy there are regional kinetics impairments present that do not have to be necessarily caught using a monoplane approach thus biasing LVEF evaluation. As the most accurate comparing to myocardial gated-SPECT as the gold standard method seems according to Bland-Altman analysis the triplane LVEF assessment that in the most accurate way simulates the 3-dimensional LV model and respects a markedly asymmetric distribution of kinetics in individual LV segments in our group of patients. Results of biplane LVEF assessment are considering their accuracy very similar to the results of gated-SPECT of myocardium. That is why a biplane LVEF measurement can be in case of a good echocardiographic image quality (a condition for enrollment into study) taking a lower time-demandingness into consideration accepted in clinical praxis for a precise oneshot LVEF assessment even in patients with LV systolic dysfunction and numerous regional kinetics impairments. A total of $91 \%$ of patients enrolled into this study had a systolic LV dysfunction. That is considerably more then in other available studies ${ }^{8}$.

The accuracy of echocardiographic measurement and mainly of triplane echocardiography is in our study unmistakably due to selection of patients with a very good echocardiographic image quality with an adequate delineation of endocardial contours. If there is a need of endocardial border extrapolation from a native 2D picture, it is possible to overcome this obstacle by use of an echocontrast agent infiltrating LV cavity and thus enhancing the visual quality. Concerning the abovementioned patient selection such an approach was in our study not needed. In summary, it can be concluded that an accuracy of echocardiographic assessment of LVEF compared to gated-SPECT of myocardium decreased from triplane to monoplane echocardiography and it was considerably determined by selection of patients with an adequate echocardiographic image quality and by a short time interval between both methods. That is why the results of LVEF measured by multiplane echocardiography using Simpson's method and by gated-SPECT of myocardium are in a group of patients with ischemic cardiomyopathy and systolic LV dysfunction mutually interchangeable.

Another situation arises in LV volumes measurement. Based on available literature there is evidence that 2-dimensional echocardiography statistically significantly correlates with gated-SPECT o myocardium. Nevertheless, in light of absolute values of results these examinations can vary markedly ${ }^{11}$. Comparing LV volumes assessment using gated-SPECT and MR the radionuclide method systematically underestimates LV volumes ${ }^{12-14}$. In a study by Omara et al. ${ }^{6}$ with consecutive pediatric patients with preserved LV systolic function and normal LV volumes echocardiography overestimated endsystolic and enddiastolic volumes. In our study, we came to an opposite finding ${ }^{6}$. Results of echocardiographic assessment of volumes do in conformity with available literature correlate with gatedSPECT of myocardium on the same quantitative level, but echocardiography significantly underestimates results of LV volumes measurement also in a group of patients with ischemic cardiomyopathy and LV systolic dysfunction and remodeled LV with increased volumes ${ }^{15,16}$. This finding can be interpreted in several ways. One can suggest that the main reason for this could be a not quite accurate geometrical tangential capture of LV in its long axis in individual apical echocardiographic projections leading into underestimation of echocardiographic measurement of LV volumes comparing to gated-SPECT. Another reason could be a lower space resolution of gated-SPECT compared to echocardiography. In the case of a regional impairment of myocardial perfusion, an endocardial contour might not be adequately visualized leading to an artificial LV cavity enlargement. The differences between individual echocardiographic approaches in LV volumes assessment were unlike LVEF measurement statistically significant. 
Above all the monoplane EDV measurement is very inaccurate (median of difference $85 \mathrm{ml}$ compared to gatedSPECT of myocardium). In a Bland-Altman analysis there was a decrease in agreement between individual methods in assessment of LV volumes from triplane to monoplane method compared to gated-SPECT of myocardium. In conclusion, echocardiography in patients with a good image quality wit a remodeled LV systematically and statistically significantly underestimates LV volumes compared to gated-SPECT of myocardium. That is why the results of these measurements are not mutually interchangeable.

\section{CONCLUSION}

Echocardiography is in the case of a good echocardiographic image quality a very precise method for one-time assessment of LVEF. In a specific group of patients with ischemic cardiomyopathy and systolic LV dysfunction, it is according to available guidelines necessary to use at least a biplanar method of discs for LVEF calculation ${ }^{17}$.

If there is a poor delineation of endocardial contours and there is a need for a precise one-time LVEF assessment, it is possible to use an alternative method of LVEF evaluation (gated-SPECT of myocardium or MR of heart). Our findings show that echocardiography systematically underestimates LV volumes compared with gated SPECT. It is a robust enough method to guide clinical management, though interchangeable use of echocardiography and SPECT should be avoided in LV volumes assessment.

\section{REFERENCES}

1. White HD, Norris RM, Brown MA et al. Left ventricular endsystolic volume as the major determinant of survival after recovery from myocardial infarction. Circulation 1987; 76: 44-51.

2. Salomon SD, Anavekar N, Skali H et al. Influence of Ejection Fraction on Cardiovascular Outcomes in a Broad Spectrum of Heart Failure Patients Circulation 2005; 112: 3738-3744.

3. Turina J, Turina M, Rothlin $\mathbf{M}$ et al. Improved late survival in patients with chronic aortic regurgitation by earlier operation. Circulation 1984; 70:I 147-52.

4. Gaasch WH, Schick EC. Symptoms and left ventricular size and function in patients with chronic aortic regurgitation. J Am Coll Cardiol 2003; 41:1325-8.

5. Sharir T, Germano G, Kavanagh PB, et al. Incremental prognostic value of post-stress left ventricular ejection fraction and volume by gated myocardial perfusion single photon emission computed tomography. Circulation 1999; 100:1035-1042.
6. Omar W, Abdel-Ghany M, Reda A. Comparison between gated SPECT and echocardiography in evaluation of left ventricular ejection fraction. Jounal of Egyptian Nat Cancer Inst 2000; 12:301306.

7. Mohan HK, Livieratos L, Gallagher S. Comparison of myocardial gated spect, planar radionuclide ventriculography and echocardiography in evaluating left ventricular ejection fraction, wall motion and thickening. J Nucl Cardiol 2004; 11(4): S25.

8. Cwajg E, Cwajg J, He ZX. Gated Myocardial Perfusion Tomography for the Assessment of Left Ventricular Function and Volumes: Comparison with Echocardiography. J Nucl Med 1999; 40:1857-1865.

9. Habash-Bseiso DE, Rokey R, Berger ChJ et al. Accuracy of Noninvasive Ejection Fraction Measurement in a Large Community-Based Clinic Clin Med Res 2005; 3(2):75-82.

10. Berk F, Isgoren S, Demir H et al. Assessment of left ventricular function and volumes for patients with dilated cardiomyopathy using gated myocardial perfusion SPECT and comparison with echocardiography. Nuclear Medicine Communications 2005; 26(8):701-710.

11. Chua T, Yin LC, Thiang TH et al. Accuracy of the automated assessment of left ventricular function with gated perfusion SPECT in the presence of perfusion defects and left ventricular dysfunction: correlation with equilibrium radionuclide ventriculography and echocardiography. J Nucl Cardiol 2000; 7 (4): 301-11.

12. Persson E, Carlsson M, Palmer J et al. Evaluation of left ventricular volumes and ejection fraction by automated gated myocardial SPECT versus cardiovascular magnetic resonance. Clin Physiol Funct Imaging 2005; 25:135-141.

13. Bavelaar-Croon C, Kayser H, van der Wall E et al. Left Ventricular Function: Correlation of Quantitative Gated SPECT and MR Imaging over a Wide Range of Values. Radiology 2000; 217: 572575.

14. Vaduganathan P, He ZX, Vick GW et al. Evaluation of left ventricular wall motion, volumes, and ejection fraction by gated myocardial tomography with technecium 99 m-labeled tetrofosmin: a comparison with cine magnetic resonance imaging. J Nucl Cardiol 1999; 6: 3-10.

15. Nakae I, Matsuo S, Koh T et al. Left ventricular systolic/diastolic function evaluated by quantitative ECG-gated SPECT: comparison with echocardiography and plasma BNP analysis. Ann Nucl Med 2005; 19(6): 447-54.

16. Vourvouri EC, Poldermans D, Bax JJ et al. Evaluation of left ventricular function and volumes in patients with ischaemic cardiomyopathy: gated single-photon emission computed tomography versus two-dimensional echocardiography. Eur J Nucl Med 2001; 28:1610-1615.

17. Lang RM, Bierig M, Devereux RB et al. Recommendations for Chamber Quantification: A Report from the American Society of Echocardiography's Guidelines and Standards Committee and the Chamber Quantification Writing Group, Developed in Conjunction with the European Association of Echocardiography, a Branch of the European Society of Cardiology. J Am Soc Echocardiogr 2005; 18: $1440-1463$. 